ENCYCLOPÉDIE Encyclopédie berbère

BERBERE

$27 \mid 2005$

27 | Kairouan - Kifan Bel-Ghomari

\title{
Khil (Khail, Khril)
}

(Ras Achakar, Cap Spartel, Maroc nord-atlantique)

L. Messili

\section{OpenEdition}

\section{Journals}

Édition électronique

URL : http://journals.openedition.org/encyclopedieberbere/1357

DOI : 10.4000/encyclopedieberbere. 1357

ISSN : 2262-7197

\section{Éditeur}

Peeters Publishers

\section{Édition imprimée}

Date de publication : 1 août 2005

Pagination : 4239-4244

ISBN : 2-7449-0538-0

ISSN : $1015-7344$

\section{Référence électronique}

L. Messili, « Khil (Khail, Khril) », Encyclopédie berbère [En ligne], 27 | 2005, document K57, mis en ligne le 01 juin 2011, consulté le 24 septembre 2020. URL : http://journals.openedition.org/

encyclopedieberbere/1357 ; DOI : https://doi.org/10.4000/encyclopedieberbere.1357

Ce document a été généré automatiquement le 24 septembre 2020.

(c) Tous droits réservés 


\section{Khil (Khail, Khril)}

(Ras Achakar, Cap Spartel, Maroc nord-atlantique)

\section{Messili}

1 Khail ou Khril*, la transcription française du toponyme arabe de ces localités - khail, ou encore khil en dialectal, signifie «cheval »-, a longtemps prêté à confusion. En fait, il s'agit bien d'El-Khail ou de Magharat El-Khail". "Magharat» (grottes, pluriel de « Mugharet ») El-Khail, car il s'agit de trois grottes creusées sur le versant nord de l'Oued Achakar (également appelé Oued Mrirat El-Khil) dont l'embouchure, située à environ trois kilomètres et demi au sud du cap Spartel (détroit de Gibraltar), délimite le relief proéminent du cap Achakar* (Camps-Fabrer H. et Daugas J.-P. 1993).

2 Ce relief correspond à une monumentale banquette projetée vers l'océan (fig. 2). Le secteur, fouillé depuis la fin du $\mathrm{xIx}^{\mathrm{e}}$ siècle, atteste d'une importante occupation préhistorique dès le Pléistocène moyen (Mugharet El-Aliya in : Doolittle H. A \&. Nahon P. 1953; Howe B. 1967). Parmi ces trois cavités, identifiées : A, B et C, la première est incontestablement la grotte d'El Khail'2, fouillée par l'équipe de l'Aspr ${ }^{3}$ (Hencken $\mathrm{H}$. 1948) et par Jodin A. (1958-1959). En 1975, Gilman A. présente une première synthèse de référence pour le secteur d'Achakar soulignant tout particulièrement l'apport des découvertes d'El Khail pour la connaissance du Néolithique local.

3 Cette hésitation entre Khail et Khril ou Khrill a été relevée par Gilman (p. 5), faisant suite, d'une part, aux travaux de l'équipe américaine du Peabody Muséum de l'Université d'Harvard dans le secteur d'Achakar, plus particulièrement dans la grotte située le plus à l'ouest de la série (Hencken H., 1947) et d'autre part, aux fouilles comparatives de Jodin dans les trois grottes (Les grottes d'El Khril, 1958-1959). Ce dernier avait bel et bien fouillé à El-Khail.

4 Il n'est plus nécessaire d'insister sur cette distinction terminologique même dans une optique historique (Gilman persévérant en ce sens, p.1094), El-Kail et El-Khril renvoyant au même toponyme autochtone. Avec les toutes dernières fouilles entreprises à El-Khail, A. Bouzouggar entérine ce consensus en nommant les grottes $\mathrm{B}$ et $C$ fouillées par Jodin, El-Khil (Bouzouggar A. et al., 2002: 209). Nous nous référerons désormais à cette orthographe de la toponymie dialectale. 


\section{Secteur géographique}

5 Située à $200 \mathrm{~m}$ au nord du cap Achakar, l'embouchure de l'Oued Achakar est dominée par des falaises de $20 \mathrm{~m}$ de hauteur. Sur le versant nord que bâtit le Djebel Achakar, celles-ci sont creusées d'une dizaine de cavités qui surmontent des talus d'éboulis situés à environ $150 \mathrm{~m}$ du rivage.

6 Les trois grottes sont perchées à 10-12 m d'altitude et leurs ouvertures distantes d'une dizaine de mètres les unes des autres. Il s'agit en fait d'un même réseau souterrain fractionné, qui débouche à différents niveaux sur la falaise. Les grottes sont cloisonnées par des effondrements de voûtes et constituent ainsi trois habitats distincts, toutefois relativement puisque les données stratigraphiques et archéologiques des remplissages concordent remarquablement Godin, 1958-59).

7 Le creusement des trois grottes correspond à la dissection du rivage au cours de l'Ouljien, étage de la mer transgressive (Tyrrhénien II, Gigout, 1949 ; Souville, 1973 : 22) qui a entamé les bancs de conglomérats des Grès de Rabat (Bourcart 1927 ;Lecointre 1952).

8 Les grottes d'El Khil ne sont, en fait, que relativement distantes de la série d'abris préhistoriques côtiers longtemps désignés comme les grottes d'Hercule. El-Khil (A) n'est qu'à $200 \mathrm{~m}$ au nord de la grotte d'El-Aliya, située à une centaine de mètres de la grotte des Idoles (versant sud du Ras Achakar). Surplombant la mer à une altitude de 20 m, El-Khil (A), la plus grande des trois, offre avec la grotte Es Saifiya (à une dizaine de mètres de la grotte des Idoles) un exemple de corrélation excellent sur les plans morphologique, stratigraphique et archéologique avec les célèbres grottes El-Aliya et grotte des Idoles, du nom des figurines en argile qu'elle a livrées.

\section{Historique des recherches}

9 Le secteur géologique a été prospecté dès la fin du XIx ${ }^{e}$ siècle (Bleicher 1875 ; Tissot $1878^{5}$ ). Les fouilles archéologiques ont été entreprises par: Buchet (1906, dans: Koehler, 1931), Michaux-Bellaire (1908), Biarnay S. et Péretié (1912) et Koehler (1931).

En 1936, l'ASPR entreprend des fouilles à Mugharet El-Aliya (Doolittle H.A. et Nahon J.-R. 1953), toutefois elles restent limitées aux niveaux néolithiques. En 1939, Carleton Coon fouille les niveaux pléistocènes. Un an plus tard, Doolittle/Nahon, et une équipe du Peabody Museum (Coon, Movius et Howe) s'installent dans le secteur dAchakar. Un rapport préliminaire sera publié en 1947.

11 Dès 1947, Hencken reprend le chantier de Mugharet El-Aliya et entame ses fouilles à ElKhil ; il prospecte, en second plan, les sites paléolithiques en plein air (Hencken 1948; Howe 1949 : 36) ; Stearns, 1950 ; Howe \& Stearns, 1955 et Howe, 1967). Cette dernière publication constitue un rapport final réunissant les découvertes récentes et les résultats de l'équipe de l'Aspr de 1947.

Enfin, les derniers ttavaux interviennent avec Jodin qui fouille systématiquement les trois abris $\mathrm{A}, \mathrm{B}$ et $\mathrm{C}$ et aboutit à une très bonne typologie du Néolithique nordmarocain, fondée sur le matériel céramique. 


\section{Stratigraphie d'El Khil}

13 Un remplissage moyen de $2 \mathrm{~m}$ d'épaisseur (2,80 $\mathrm{m}$ à El-Khil (A)) a montré, d'après l'ASPR, la succession de 8 niveaux qui couvrent des temps d'occupation allant du Néolithique ancien aux temps historiques et actuels (Gilman, p. 12, fig. 5 ; p. 98) :

A : sables gris (actuel)

B : sables argileux, gris plus foncé que A

C : comparable à $\mathrm{A}$

D : mêmes sédiments, plus foncés, renfermant de nombreux mollusques

$\mathbf{F}$ : graviers dans une matrice compacte

$\mathrm{H}$ : sédiments gris très foncé

14 Jodin (1958-1959) distinguait 4 niveaux archéologiques surmontant le socle rocheux de la grotte, lui-même situé à $11 \mathrm{~m}$ au-dessus du niveau de la mer :

C I : sables récents

C II : $\mathbf{a b}$ : sédiment noir ( $\mathbf{a}$ et $\mathbf{b}$, séparés par une croûte calcaire, constituent deux souscouches à El-Khil B et C)

$\mathrm{C}$ : niveau brun foncé (à El-Khil (A) seulement)

C III : sédiment argileux jaunâtre avec blocs d'éboulis (stérile à El-Khil B et C)

C IV : sédiment sableux jaunâtre stérile.

Les deux stratigraphies ne concordent pas parfaitement, les différences étant certainement dues à la nature et au suivi des fouilles. Hencken relève que le contexte est le même sur $50 \mathrm{~cm}$ de dépôts, du niveau $\mathrm{F}$ à $\mathrm{H}$. Pour les niveaux correspondants, $\mathrm{C}$. III et C. II (b), respectivement, Jodin distingue un niveau renfermant du matériel paléolithique (faune et industrie), du niveau sus-jacent, datant du Néolithique ancien. La position stratigraphique de la céramique cardiale correspond au niveau le plus ancien d'un Néolithique qui repose directement sur le Paléolithique moyen. Les récents travaux de Bouzouggar à El-Khil B et $\mathrm{C}$ ont livré une datation C14 de $5720 \pm 150$ ans BP (charbons de bois) pour ce niveau Néolithique ancien.

16 L'absence d'un épisode épipaléolithique a été soulignée dans l'ensemble du secteur et en grottes, par opposition aux sites en plein air, toutefois elle pourrait coïncider avec une situation paléogéographique différente de celle installée durant le Néolithique. Les différentes exploitations des ressources, marines près du rivage, terrestres vers l'intérieur des terres (Cahf Taht El-Ghar), pourraient témoigner de différentes modalités d'occupation (Gilman 1975, Bouzouggar et al, 2002).

\section{Le matériel archéologique}

17 Ces niveaux permettaient de suivre en détail l'évolution des caractères typologiques de la céramique : cardiale, incisée puis lisse au cours de l'Age de Bronze. La stratigraphie comparée des trois grottes concorde, à de faibles variations près. Elle fait ressortir deux traits marquants. D'une part, une excellente évolution de la céramique est mise à évidence. D'autre part, la décadence de l'outillage lithique, les catégories ne variant pas sensiblement (Gilman A., p. 78) : elles évoluent d'une prédominance des pièces à dos (éclats et lames) vers une prédominance des lamelles à dos, des pièces à retouches continues, des pièces à coches et des microlithes géométriques. À partir du Néolithique récent, l'outillage devient beaucoup moins spécialisé. 
trairement à la grotte des Idoles, celles d'El-Khil n'ont livré que quelques outils en os. Par contre, le mobilier « rituel » est proche sur deux plans : celui de l'utilisation de l'ocre, attestée sur des tessons de céramique ainsi que sur de nombreux autres supports, et l'incontestable fabrication de figurines en terre cuite. Les fouilles ont mis au jour une figurine semblable aux 47 figurines inédites découvertes, 50 ans plutôt, par Buchet dans la grotte des Idoles (Camps-Fabrer, 1966, Messili 2001). L'usage des coquillages pour la parure est inexistant, contrairement à ce qu'il en est dans ce second site.

à El-Khil, par opposition à la céramique lisse, à engobe rouge et polie des niveaux récents, types qui persistent d'ailleurs longtemps. Il distingue un Néolithique ancien d'un Néolithique récent que caractérise la céramique de type Achakar (p. 76). Pour le premier, à côté des céramiques cardiales et cannelées majoritaires $(45 \%)$, d'autres types sont présents : céramique incisée, céramique à décor de stries, céramique à décor de cordons et céramique à impressions digitales. Pour le Néolithique récent, la céramique rouge dite "d'Achakar ", la céramique à décor au peigne et à impression sont majoritaires.

21 Ce schéma est similaire dans la grotte d'El-Aliya. Les travaux de Hencken ont mis en évidence dans les niveaux néolithiques plusieurs types de céramique à décor cardial, cannelé, incisé, à cordons et quelques tessons de céramique fine à engobe rouge ou noir. Les fouilles de Tarradell à Caf Taht El-Ghar ont révélé, de leur côté, la présence d'une céramique campaniforme attestant des rapports avec l'Espagne. Dans la grotte des Idoles, la présence de quelques spécimens de cette dernière, atteste d'échanges et de mouvements de populations.

Il va sans dire que la contribution des données d'El Khil à l'établissement d'une typologie (aussi bien sur matériel lithique que céramique) du Néolithique dit d'Achakar est due en grande partie à la qualité des fouilles offertes, celles de l'équipe de I'Aspr entte autres. Gilman qui en a présenté la synthèse soulignait déjà le caractère peu ou pas exploitable des fouilles de Jodin.

De récents travaux sont entrepris par une équipe belgo-marocaine à El-Khil (Bouzouggar et al., 2001 et 2002). Il reste à espérer de nombreux résultats issus de fouilles systématiques, continues et multidisciplinaires, pour envisager la révision des anciens travaux. 


\section{BIBLIOGRAPHIE}

BIARNAY S. et PÉRETIÉ A., 1912. « Recherches archéologiques au Maroc. La caverne des Idoles au cap Spartel ». Archives marocaines, vol. 18, pp. 373-400.

BLEICHER G, 1875. «Recherches d'Archéologie préhistorique dans la Province d'Oran et dans la partie occidentale du Maroc ». Matériaux pour l'Histoire Primitive et Naturelle de l'Homme. Toulouse, $2^{\mathrm{e}}$ série, vol. 6, pp. 193-212.

BOURCART J., 1927. « Premiers résultats d'une étude du Quaternaire du Maroc ». Bulletin de la Société Géologique de France, 4 e série, vol. 27, pp. 3-33.

BOUZOUGGAR A., KOZLOWSKI J.K. et OTTE M., 2002. « Étude des assemblages lithiques atériens de la grotte d'El Aliya à Tanger (Maroc) ». L'Anthropologie, vol. 106, n² 2, pp. 207-248.

BOUZOUGGAR A., OTTE M., ATKI H., BEN HADI S., BRUTOUT T., DERCLAY C, KAOUANE C, MARGAA A., MILLER R., MOHIB A., MOUSHINE T., NAMI E., NOIRET P., WRINN T., 2001. « Nouvelles découvertes archéologiques dans la région de Tanger ». XIV Congrès international de l'UISPP, Pré-actes, Liège, 2-8 septembre 2001, pp. 336-337.

CAMPS G, 1984. « C42. Achakar ». Encyclopédie berbère, I, pp. 107-110.

CAMPS-FABRER H., 1966. « Matière et art mobilier dans la Préhistoire nord africaine et saharienne ». Mémoires du CRAPE, vol. 5, A.M.G, Paris.

CAMPS-FABRER H. et DAUGAS J.-P, 1993. « C25. Cardiale ». Encyclopédie berbère, t. 12, pp. 1777-1779.

DOOLITTLE H. et A. NAHON P., 1953. « The caves of Hercules ». Tinga, Tangier, $\mathrm{n}^{\circ} 1$.

FALLOT P. et REY M., 1953. « Les formations gréseuses du Cap Spartel ». Notes et Mémoires, Service Géologique, Maroc, t. VII, n 117, pp. 73-87.

GIGOUT M., 1949. « Définition d'un étage ouljien ». Comptes Rendus de l'Académie des Sciences, Paris, t. 229, pp. 551-555.

GILMAN A., 1975. « The Later Prehistory of Tangier. Morocco ». American School of Prehistoric Research, Peabody Museum of Anthropology and Ethnology, Harvard Univer-sity, $\mathrm{n}^{\circ} 23$.

HENCKEN H., 1949. « The prehistoric Archaeology of the Tangier Zone, Morocco ». Proceedings of the American Philosophical Society, vol. 92, $\mathrm{n}^{\circ}$ 4, 1948, pp. 282-288.

HOWE B., « A program of excavations in the Stone Age of Northwest Africa ». Archaeology, vol. 2, pp. 76-83.

HOWE B., 1967. « The Palaeolithic of Tangier, Morocco. Excavations at Cape Achakar, 1939-1947 ». Bulletin of the American School of Prehistoric Research, $\mathrm{n}^{\circ} 22$.

HOWE B. et Movius H.L.Jr., 1947. « A Stone Age cave site in Tangier. Preliminary report on the excavations at the Mugharet El-Aliya, or High cave, in Tangier ». Papers of the Peabody Muséum of American Archaeology and Ethnology, Harvard University ; Cambridge, Massachusetts, vol. XXVIII, $\mathrm{n}^{\circ} 1$, pp. 6-7.

HOWE B., et STEARNS CE., 1955 (1953). « Geology and Archaeology of Cape Ashakar, Tangier, Morocco ». Actas del Congreso Arqueolôgico del Marruecos Español, Tetuán, pp. 39-51.

KOEHLER H., 1931. « La grotte d'Achakar au Cap Spartel ». Etudes de Préhistoire Marocaine, Collection Marrochitana, $\mathrm{n}^{\circ} 1$. 
JODIN A., 1958-59. «Les grottes d'El Khril à Achakar ». Bulletin d'Archéologie Marocaine, Tanger, t. III, pp. 249-313.

JOLEAUD L., 1926. «Contribution à l'étude du Quaternaire dans le Maroc atlantique ». Ext. du C.R.S. de la Société Géologique de France, Paris, n 16 ; pp. 156-159.

LECOINTRE G, 1952. « Recherches sur le Néogène et le Quaternaire marins de la côte occidentale du Maroc ». Notes et Mémoires, Service géologique, Maroc, vol. 99,t. I.

MESSILI L., 2001. Figurines en argile modelée cuite de la grotte des Idoles, Achakar, Cap Spartel (Maroc), collection préhistorique du musée de l'Homme (Paris), réévalution. Mémoire de DEA, IPH, Paris. MiCHAUX-BELLAIRE E., 1908. « Fouilles dans la nécropole romaine de Tanger ». Revue du Monde Musulman, vol. 6, pp. 419-432.

MOULIÉRAS A., 1899. « Le Maroc inconnu. Étude géographique et sociologique ». Tome I : « Exploration du Rif(Maroc septentrional) », tome II : « Exploration des Djebala (Maroc septentrional) ». Augustin Challamel éditeur, Paris.

RENÉ-LECLERC C, 1907. « Le Maroc septentrional : souvenirs et impressions (été 1904) ». Imprimerie algérienne Alger-Mustapha, Alger.

SOUVILLE G, 1973. « Atlas préhistorique du Maroc, tome 1 : Maroc atlantique ». Éditions CNRS, Paris.

STEARNS CE., 1950. « The Pleistocene Geology of the Atlantic coast of Northern Morocco ». Bulletin of the Geological Society of America, Baltimore, vol. 39.

TARRADEL M., 1954. « Avance de la Primera Campaña de excavaciones en Caf Taht El-Ghar ». Tamuda, t. II, p. 307.

TARRADEL M., 1955. « Noticia sobre la excavación de Gar Cahal ». Tamuda, t. II, pp. 344-356.

TISSOT C, 1878. « Recherches sur la géographie comparée de la Mauritanie tingitane ». Mémoires présentés à l'Académie des Inscriptions et des Belles-lettres de l'Institut de France, $1^{\mathrm{re}}$ série, Paris, t. 9, pp. 139-322.

\section{NOTES}

1. Le nom donné aux sites semble inspiré de l'usage millénaire des lieux, comme étables et bergeries, par les habitants locaux.

2. La grotte $\mathrm{A}$, la plus grande des trois : 10,20 m de profondeur, est située bien à l'ouest, son ouverture tournée vers la mer. La disposition interne de véritables chambres a dû constituer un avantage pour son habitabilité.

3. American School of Prehistoric Research.

4. "At Ashakar where most of our information comes from Mugharet El-Khail, El-Khril and Grotte des Idoles, the later Neolithic is distinguished by the presence of Ashakar ware, both decorated and redpolished» (p. 109).

5. "Nous avons constaté, au contraire, dans le Djebel Achakar, trois autres cavités naturelles qui ne sont guère connues, il est vrai, que des chasseurs de sangliers, mais qui ne peuvent prétendre à l'honneur de représenter le Specus Herculis. Deux de ces grottes s'ouvrent dans une des gorges orientales du Djebel ; la troisième, remarquable par sa large ouverture de forme triangulaire, s'enfonce sous une des falaises du versant occidental, située entre le Cap Spartel et le golfe de Tanger. Il est très possible, en outre, que d'autres cavernes du même genre aient existé dans cette partie du littoral sans qu'il en reste aucune trace aujourd'hui» (p. 189). 
INDEX

Mots-clés : Maroc, Néolithique, Paléolithique, Préhistoire 